\title{
Treating statin-intolerant patients
}

This article was published in the following Dove Press journal:

Diabetes, Metabolic Syndrome and Obesity:Targets and Therapy

27 April 2011

Number of times this article has been viewed

\section{Marcello Arca \\ Giovanni Pigna}

Atherosclerosis Unit, Department of Internal Medicine and Allied Medical Specialities, Sapienza University of Rome, Rome, Italy
Correspondence: Marcello Arca Dipartimento di Medicina Interna e Specialità Mediche, Sapienza Università di Roma,Azienda Policlinico Umberto I,Viale del Policlinico, 155, 00161, Rome, Italy

Tel +3906 445I 354

Fax +39064463534

Email marcelloarca@libero.it
Abstract: Statins are effective in reducing cardiovascular events and are safe for almost all patients. Nevertheless, intolerance to statins is frequently faced in clinical practice. This is mostly due to muscular symptoms (myalgia with or without increase of plasma creatinine kinase) and/or elevation of hepatic aminotransferases, which overall constitutes approximately two-thirds of reported adverse events during statin therapy. These side effects raise concerns in patients as well as in doctors and are likely to reduce patients' adherence and, as a consequence, the cardiovascular benefit. Therefore, it is mandatory that clinicians improve their knowledge on the clinical aspects of muscular and hepatic side effects of statin therapy as well as their ability to manage patients with statin intolerance. Besides briefly examining the clinical aspects and the mechanisms that are proposed to be responsible for the most common statin-associated side effects, the main purpose of this article is to review the available approaches to manage statinintolerant patients. The first step is to determine whether the adverse events are indeed related to statin therapy. If so, lowering the dosage or changing statin, alternate dosing options, or the use of nonstatin compounds may be practical strategies. The cholesterol-lowering potency as well as the usefulness of these different approaches in treating statin-intolerant patients will be examined based on currently available data. However, the cardiovascular benefit of these strategies has not been well established, so their use has to be guided by a careful clinical assessment of each patient.

Keywords: statin therapy, atorvastatin, rosuvastatin, aminotransferase levels, myopathy

\section{Introduction}

The 3-hydroxy-3-methylglutaryl coenzyme A (HMG-CoA) reductase inhibitors, or statins, are the mainstay of lipid-lowering therapy because of their well-established efficacy for reducing cardiovascular disease (CVD) morbidity and mortality in various at-risk populations. ${ }^{1}$ In general, statin therapy is associated with rare occurrences of serious adverse events and is considered to be safe. ${ }^{2,3}$ Nevertheless, a significant proportion of subjects taking these drugs may experience some degree of intolerance. In particular, statin-induced myopathy (SIM) is by far the most common side effect. A less common side effect of statin therapy is the increase of serum aminotransferase levels, which is considered the manifestation of hepatic toxicity. ${ }^{4}$

Despite the fact that these adverse effects are reversed after treatment withdrawal, many patients with an indication for statins refuse therapy because of concerns about muscle or liver toxicity. This may represent a significant barrier to maximizing cardiovascular risk reduction for many patients with dyslipidemia. Therefore, a better understanding of the relatively common statin-related adverse effects may improve the 
clinician's ability to manage patients with these problems. In this review, after briefly recapitulating incidence data and mechanisms whereby statins may cause muscle-related or hepatic toxicity, we will examine management strategies for patients who are intolerant to statins due to these common adverse effects.

\section{Clinical aspects and mechanisms of statin-associated adverse effects}

Several studies have evaluated the incidence of adverse events during statin therapy. In a meta-analysis of over 70,000 subjects in 18 primary and secondary prevention placebo-controlled trials, the number needed to harm (NNH) for any adverse event with statins was 197 versus 27, which was the number needed to treat (NNT) to prevent one cardiovascular event. ${ }^{5}$ In other words, treating 1,000 patients would prevent 37 cardiovascular events and cause 5 adverse events. In this analysis, serious adverse events, such as creatine kinase $(\mathrm{CK})>10$ times upper limit of normal (ULN) or rhabdomyolysis, are rare and have a NNH of 3,400. Rhabdomyolysis alone was extremely rare with an $\mathrm{NNH}$ of 7,428. In the search for differences between statins, this study showed that fluvastatin, the least efficacious, had the lowest rate of adverse events, and atorvastatin, the most efficacious, had the highest rate. Simvastatin, pravastatin, lovastatin, and rosuvastatin appeared to have similar rates of adverse events. In a systematic review of 20 clinical trials, Law and Rudnicka ${ }^{6}$ reported that the incidence of myopathy and minor muscle pain incidence was 195 cases per 100,000 patient-years (95\% confidence intervals [CI]: -38 to 410 ). The incidence of rhabdomyolysis was 1.6 cases per 100,000 patient-years (95\% CI: -2.4 to 5.5 ). However, it must be noted that the frequency with which clinicians encounter SIM in real-world clinical practice is often much higher than that reported in clinical trials. One likely explanation for this discrepancy is that the rate of myopathy in clinical trials is artificially underestimated because patients at increased risk for statin-induced adverse effects tend to be excluded prior to randomization. ${ }^{7}$ Also, many patients in clinical practices may not be as healthy as those enrolled in clinical trials and often have more severe comorbidities.

Data on the real-world incidence of SIM may be available through several drug side-effect reporting systems. For example, in the Food and Drug Administration's (FDA) Adverse Event Reporting System database recorded until 2002, the reporting rates per million statin prescriptions was 0.38 cases for myopathy and 1.07 cases of rhabdomyolysis. ${ }^{8}$ However, this source might produce biased information due to the fact that the event reporting is voluntary and thus may have resulted in underreporting of adverse effects. To obtain data that are representative of the clinical practice, it may be helpful to examine databases from cohort studies or from closed systems such as managed care organizations. The Prediction of Muscular Risk in Observational Conditions (PRIMO) produced one of these databases. ${ }^{9}$ In the PRIMO study, over 7,900 hyperlipidemic patients treated with highdose statin therapy were enrolled in a 12-month, prospective observational follow-up. Muscle symptoms were reported by $11 \%$ of patients. This figure has been confirmed by others, ${ }^{10}$ so we can reasonably state that SIM may affect $10 \%-15 \%$ of statin users.

The clinical presentation of statin myopathy varies from mild fatigue to rhabdomyolysis requiring hospitalization. The most frequently reported symptoms include myalgia, fatigue, weakness, generalized aching, and low back or proximal muscle pain..$^{2,11,12}$ There have been less frequent complaints of tendon pain and nocturnal muscle cramps. ${ }^{11}$ According to well accepted definitions, myalgia is defined as muscular symptoms without $\mathrm{CK}$ elevations; myositis refers to muscle symptoms with CK elevation; and rhabdomyolysis is defined as muscle symptoms with marked CK elevations ( $>10$ times ULN) with an elevated plasma creatinine and the occasional presence of brown urine. ${ }^{12}$

The temporal relationship between initiation of statin treatment and onset of symptoms is widely variable, as is the time between cessation of statin treatment and the resolution of symptoms. In a study that used two large UK primary care databases covering an active population of about 5 million people and including many patients with a follow-up period of over ten years, it has been reported that most SIM cases occur within the first 12 weeks of statin exposure, but few can be seen up to 52 weeks of treatment. ${ }^{13}$ SIM does not appear to be related to statin dosage. In a review of several atorvastatin trials, treatment-related myalgia occurred at a similar rate of $1.4 \%$ and $1.5 \%$ in subjects receiving 10 or $80 \mathrm{mg}$ of atorvastatin compared with a rate of $0.7 \%$ with placebo. ${ }^{14}$ A retrospective analysis of safety from the PROVE-IT trial also suggested that statin adverse effects are not related to low density lipoprotein (LDL) level. ${ }^{15}$ In fact, muscular and hepatic side effects were found to occur at the same rate across all on-treatment LDL-cholesterol (LDL-C) levels, including very low levels of $40 \mathrm{mg} / \mathrm{dL}$. This phenomenon has been confirmed in a recent meta-analysis comparing different statin doses and on-treatment LDL-C levels. ${ }^{16}$ Although the exact mechanisms causing SIM has not been determined, several hypotheses have been proposed. It has 
been suggested that cholesterol reduction with statins may perturb the integrity of the plasma membrane of myocytes because cholesterol plays a key role in cell membrane fluidity. ${ }^{17}$ Others have proposed that statins induce myopathy by favoring the deficiency of coenzyme Q10 (CoQ10), which is a metabolite of the HMG-CoA reductase pathway. ${ }^{18}$ CoQ10 plays a key role in the electron transport chain, and a reduction in this coenzyme could result in an abnormal mitochondrial respiratory function. However, several lines of evidence make these explanations unlikely. Firstly, when cholesterol is decreased by inhibiting squalene synthetase, no increase in myotoxicity is observed. ${ }^{19}$ Human and animal studies have demonstrated that statin treatment may reduce serum CoQ10 levels; however, myocyte CoQ10 levels have not been consistently decreased with statin treatment. ${ }^{20}$

Another possible explanation of SIM relates to the observation that statins induce the apoptosis or programmed cell death of myocytes by reducing isoprenoids levels. Isoprenoids are lipids produced by HMG-CoA reductase pathway. ${ }^{21}$ Isoprenoids are linked to proteins by a process known as farnesylation. According to this theory, statins block the production of farnesyl pyrophosphate and this prevents the prenylation of GTP-binding proteins Ras, Rac, and Rho. A reduction in the levels of the prenylated forms of these proteins leads to increased cytosolic calcium levels with subsequent activation of the proteolytic enzymes capsase-3 and capsase-9, which have a central role in cell death. This theory is also supported by an in vitro study demonstrating that statin-induced apoptosis of muscle cells is prevented by supplementation with the isoprenoids farnesyl pyrophosphate and geranylgeranyl pyrophosphate but not CoQ10. ${ }^{22}$ Finally it has been proposed that statins impair intracellular calcium homeostasis by interfering with the mitochondrial respiratory chain and by affecting ryanodine receptor one (RyR1), which pumps calcium into the cytoplasm. Increased cytoplasmic calcium levels have been shown to cause cramps, myalgias, and apoptosis. ${ }^{23,24}$

Recently, genetic risk factors for statin myopathy have been identified. Investigators from the ongoing Study of the Effectiveness of Additional Reductions in Cholesterol and Homocysteine (SEARCH) trial hypothesized that strong associations might exist between treatment with a high-dose statin regimen and genetic variants that affect statin blood levels. ${ }^{25}$ In this trial, a genome-wide analysis demonstrated that myopathy was strongly associated with a single nucleotide polymorphism within intron 11 of SLCO1B1 on chromosome 12. SLCO1B1 is the gene that encodes the organic anion transporting polypeptide responsible for hepatic uptake of statins. In the SEARCH, $60 \%$ of the cases of myopathy were associated with $S L C O 1 B 1$ variants.

The most commonly encountered hepatic biochemical abnormality during statin therapy is the asymptomatic elevation of aspartate aminotransferase (AST) and alanine aminotransferase (ALT) which appears to be a class effect of statins. ${ }^{2,4}$ This has been also defined as 'transaminitis', in which liver enzymes are elevated in the absence of clear hepatoxicity. This condition is usually transient with full resolution following withdrawal of the drug, although this may take several months. It is rare for statins to cause isolated elevations in gamma-glutamyl transferase (GGT). ${ }^{26}$ Several reports indicate that the occurrence of aminotransferase elevation during statin therapy ranges from $1 \%-3 \% .^{27,28}$ This effect appears to be dose related ${ }^{29,30}$ and hence may be related to bioavailability. In a recent meta-analysis, ${ }^{16}$ it has been shown that atorvastatin $80 \mathrm{mg}$ and simvastatin $80 \mathrm{mg}$ are associated with a persistent elevation of ALT ( $>3$ times ULN) up to 5 times compared to atoravastatin $10 \mathrm{mg}$ and simvastatin $20-40 \mathrm{mg}$ ( $0.2 \%$ vs $1.0 \%)$. Hepatocellular injury seen during statin therapy seems to be an early side effect as demonstrated in several statins trials where AST and ALT elevation appeared in the initial 3 months of treatment. ${ }^{31}$ Liver-related symptoms occurred on average 4 weeks (range 1 to 8 weeks) after initiation of treatment but resolved within 4 weeks of statin therapy discontinuation.

The mechanism by which statins may induce hepatocellular injury is unclear. Animal studies have suggested that the depletion of mevalonate or one of its sterol metabolites caused by the inhibition of 3-hydroxyl, 3-methyl-glutaryl-CoA reductase ( $\mathrm{HMG}-\mathrm{Co} \mathrm{A}$ ) enzyme may be responsible for the elevated liver enzymes. ${ }^{32}$ It has been suggested that the inherent metabolic characteristics of statins may have some relevance. In fact, the different statins have distinctly different metabolic pathways, as simvastatin, lovastatin, fluvastatin, and atorvastatin are metabolized by cytochrome P450 system, whereas pravastatin, rosuvastatin, and pitavastatin undergo minimal hepatic metabolism. Moreover, statins vary in their degree of lipophilicity, which may have an impact on their likelihood of being associated with aminotransferase elevations. The meta-analysis by Dale et $\mathrm{al}^{33}$ demonstrated that the less lipophilic statins (pravastatin, rosuvastatin, atorvastatin, fluvastatin) increased the relative risk of aminotransferase elevation compared to the more lipophilic ones (lovastatin, simvastatin, cerivastatin). In this regard, it is interesting to note that the opposite has been observed for CK elevation. ${ }^{12,13}$ A clear explanation for this is not available even though one could implicate the hepatic organic 
anion transport protein (OATP or $S L C O 1 B 1$ ) that plays an important role in facilitating the penetration of statin into the hepatocytes. It has been reported that genetic variations in SLCO1B1 have a larger effect on the area under the plasma concentration-time curve of atorvastatin than that observed with the more hydrophilic rosuvastatin. ${ }^{34}$

The natural history of elevated liver enzymes due to the long-term use of statins is poorly understood. However, it is recognized that in some individuals, this elevation is transient and may be physiological rather than pathological and that some patients display 'adaptation', where liver enzymes stabilize/normalize if the drug is not withdrawn. ${ }^{35}$ There are no studies that correlate hepatic histology with elevations in liver enzymes to differentiate between true hepatotoxicity and an adaptive process. The US National Lipid Association's (NLA) Safety Assessment Task Force concluded in 2006 that there was no evidence of a relationship between elevated transaminases, statin therapy, and risk of significant liver injury. ${ }^{4}$ Furthermore, they also concluded that routine monitoring of liver enzymes did not identify those individuals at risk of developing idiosyncratic liver failure. In addition, recent evidence suggests that moderate elevation of transaminases should not contraindicate the initiation of statin therapy. A post-hoc analysis of the secondary prevention Greek Atorvastatin and Coronary Heart Disease Evaluation (GREACE) study, ${ }^{36}$ assessed the cardiovascular and liver outcomes in a total of 437 patients presenting moderately elevated liver enzymes ( $<3$ times ULN) at enrollment, possibly associated with non-alcoholic fatty liver disease. Two hundred twentyseven of these individuals who were treated with a statin (mainly atorvastatin, $24 \mathrm{mg}$ per day) had substantial improvement in liver tests $(P<0.0001)$, whereas the 210 individuals not treated with a statin had further increases of liver enzyme concentrations during the 3-year follow-up of this study. Furthermore, patients with abnormal liver tests who received a statin experienced fewer cardiovascular events in comparison to patients with abnormal liver tests who did not receive one (68\% relative risk reduction, $P<0.0001)$. Interestingly, this cardiovascular benefit was greater $(P=0.0074)$ than it was in patients with normal liver enzymes.

The most frequently seen histological appearance of statin-induced liver injury is inflammation of the portal tracts with mild piecemeal necrosis and focal periportal fibrosis. ${ }^{37}$ As serious hepatotoxicity caused by statins is rare, these findings are seldom seen. The FDA's Adverse Event Reporting System database until 2004, reported a rate of 0.69 cases of liver failure/hepatitis per million statin prescriptions, a figure similar to that reported for liver failure/hepatitis in the general adult population. ${ }^{4}$ Analysis of an administrative database showed 6.1 to 12.8 hepatic events per 10,000 person-years of hospitalized patients on statins. ${ }^{38}$ None were hospitalized within 6 months of starting their statin. Furthermore, only 1 of the 51.741 patients who underwent liver transplantation between 1990 and 2002 was taking a marketed statin. ${ }^{27,39}$

However, recent literature indicates that potential remains for these more serious hepatotoxic reactions in association with statins. Adverse drug reaction reports from the UK Committee on Safety of Medicines show four deaths caused by atorvastatin-induced hepatotoxicity over an eight-year period ( 0.5 deaths per annum). ${ }^{40}$ In addition, there are also reports of rosuvastatin, fluvastatin, and atorvastatin inducing or revealing autoimmune disease, including autoimmune hepatitis. This is an extremely rare effect and there is evidence that the hepatic effect may be reversible when the drug is withdrawn. ${ }^{41-43}$ Finally, in very rare circumstances, statin therapy may cause liver failure. ${ }^{44}$ Overall, the long-term hepatic safety of statins is reassuring. It has been, in fact, reported that 24 million years of patient treatment with lovastatin reveal a rate of acute liver failure of 1 per 1.14 million patient-treatment years, which is similar to the rate of idiopathic acute liver failure. ${ }^{37}$ Nevertheless, the potential for statin-associated severe liver injury makes the monitoring of liver enzymes during this treatment important to recognize drug-induced liver injury as early as possible.

\section{Strategies for managing statin intolerant patients}

The first step in the management of intolerance to statins is to rule out any possible conditions that increase the risk of developing SIM or aminotransferase elevations. A list of the most common of these conditions is reported in Table 1. The National Lipid Association Statin Safety Task Force has provided recommendations for the management of musclerelated symptoms in patients receiving statin therapy ${ }^{4}$ and these are incorporated in Figure 1. In summary, in patients with moderate symptoms and without significant CK elevation $(<5 \times \mathrm{ULN})$, progress can be followed clinically. On the other hand, in patients with severe symptoms and in those with $\mathrm{CK}$ elevated more than $5 \times \mathrm{ULN}$, statins should be stopped. Once CK is normalized, patients should be rechallenged with the same statin at the same dosage. Otherwise, different approaches can be considered (Table 2). The use of agents (coenzyme Q10 and vitamin D preparation) to alleviate muscular symptoms has also been proposed.

Guidelines have also been issued to manage liver intolerance to statins, ${ }^{4,26,27}$ and these are summarized in Figure 2. Baseline 
Table I Potential risk factors for statin-induced myopathy (SIM) and hepatic side effects of statins

\begin{tabular}{ll}
\hline Statin-induced myopathy & Hepatic toxicity \\
\hline Frailty and low body mass index & Acute viral diseases \\
Advanced age ( $>80$ y) & Alcohol-associated liver diseases \\
High physical activity & Advanced chronic liver diseases \\
Heavy alcohol consumption & Mildly lipophilic statins \\
Drugs affecting statin metabolism & Genetic factors (CYP450 \\
(gemfibrozil, cyclosporin, amiodarone, & isoenzymes) \\
macrolides antibiotics, verapamil, & \\
systemic use of azole antifungale, & \\
warfarin, protease inhibitors) & \\
Renal insufficiency & \\
Hypothyroidism & \\
Rheumatic diseases & \\
Metabolic muscle diseases & \\
Major surgery & \\
Genetic factors (CYP450 variants, & \\
drug transporter variants) &
\end{tabular}

elevations of hepatic transaminases $<3$ times ULN are not a contraindication to statin therapy. Many patients with diabetes, metabolic syndrome, or obesity have nonalcoholic fatty liver disease with transaminase levels fluctuating between 1.5 and $3 \times$ ULN $^{29}$ After establishing that no other etiologies are responsible for the transaminase elevations, a statin at a low-to-moderate dose can be started while monitoring alanine aminotransferase levels. No consensus exists regarding the best time to recheck liver biochemistry values. In the HPS and in the AFCAPS/TexCAPS trial, the liver profile was rechecked after 3 and 2 weeks, respectively, with normalization of the values in more than $70 \%$ of the cases. Other authors suggest repeating the test after 6 weeks. ${ }^{45}$ In cases where the increment in aminotransferase levels is $>3 \times \mathrm{ULN}$, it is recommended to stop the treatment and reassess liver tests. After that, rechallenging the patient with the same drug and dose, a different statin, or the same statin at a lower dose should be attempted. ${ }^{26,27}$ The ideal time period between the normalization of the liver enzyme levels and the initiation of the rechallenge is not well-specified. Conversely, when the elevation in aminotransferase levels is persistent after statin withdrawal or reoccurs after a statin rechallenge, other options should be considered. Several authors have recommended using low-dose statin treatment because of the possible greater incidence of liver enzyme elevations with higher doses. It has also been proposed that liver biochemistry monitoring should be performed every month for the first 3 to 4 months and four times a year thereafter. Additionally, the use of statins, which are not metabolized by the liver or the

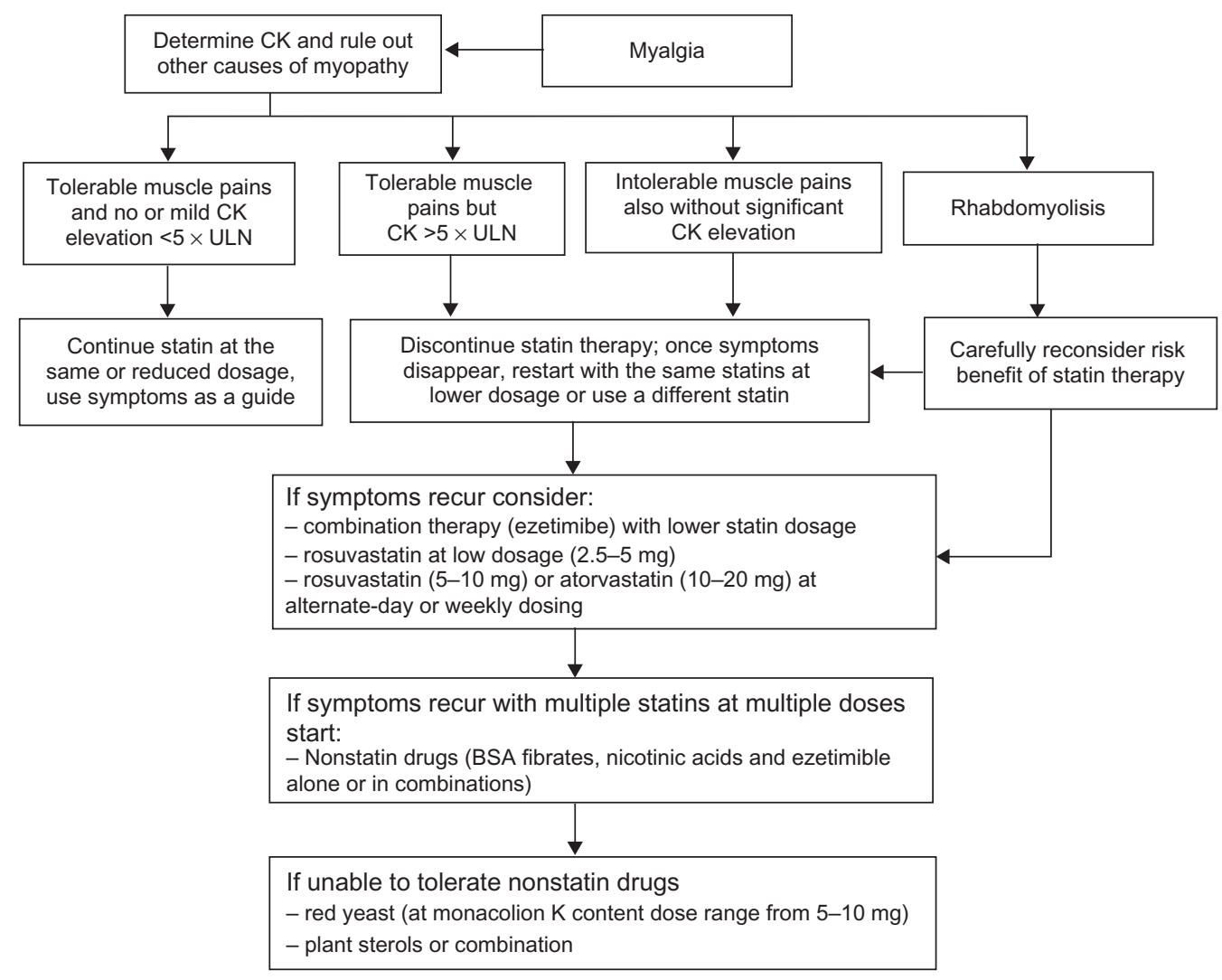

Figure I Algorithm for management of statin induced myopathy.

Abbreviations: BSA, bile acid sequestrants; CK, creatine kinase; ULN, upper limit of normal. 
Table 2 Possible approaches to manage statin intolerant patients

- Switching to another statin (preferably with different metabolism)

- An alternate-day or weekly dosage of statins with longer half-life

- Combination therapy (with ezetimibe) with infrequent statin dosing

- Nonstatin lipid lowering drugs (BSA ezetimibe, fibrate, nicotinic acid)

- Dietary manipulation and nutraceuticals

Abbreviation: BSA, bile acid sequestants.

use of nonstatin compounds can be considered. A detailed evaluation of the different options to manage statin intolerant patients is reported below.

\section{Switching to another statin}

Data demonstrating the usefulness of switching intolerant patients to another statin are scarce. Hansen et al, ${ }^{46}$ in a follow-up of 45 patients with confirmed SIM, found that 37 of them received another statin. Twenty-one patients $(57 \%)$ reported recurrent muscle pain, whereas 16 (43\%) tolerated the new statin without reporting symptoms. These data demonstrate that the recurrence of symptoms is very common on rechallenge and indicate that this strategy may be useful in only some patients. The criteria to select the new statin are not well-defined. One possibility could be to change from a

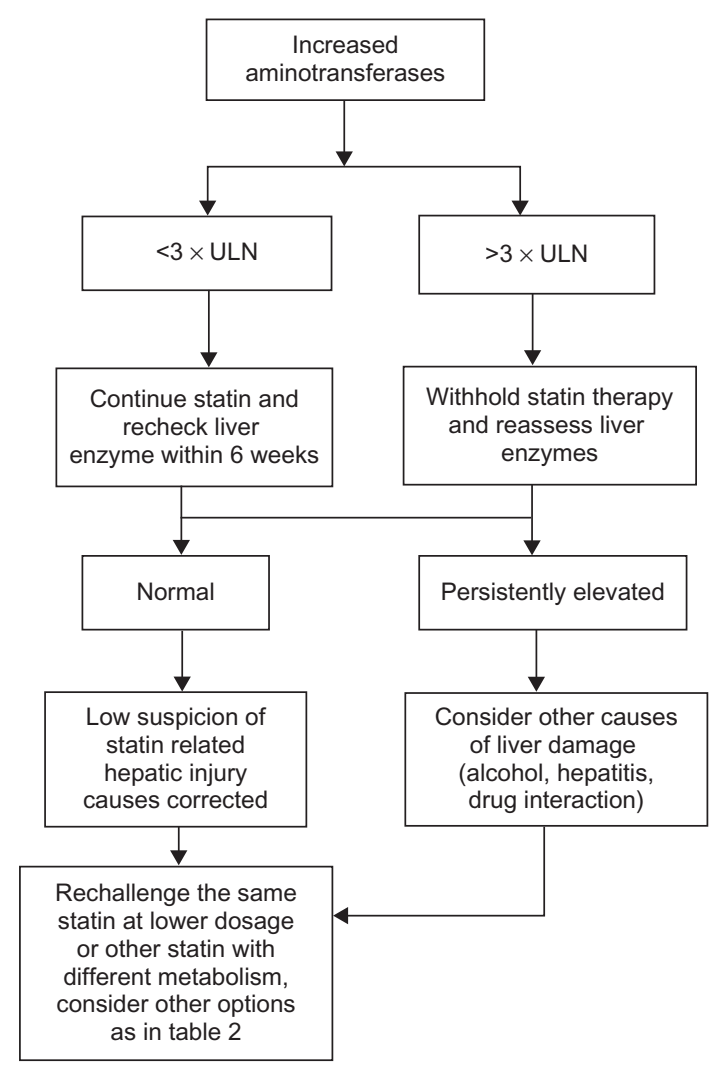

Figure 2 Algorithm for management for abnormal liver enzymes during statin therapy.

Abbreviation: ULN, upper limit of normal. mildly to a highly lipophilic statin or from a P450-dependent to a non-P450-dependent statin.

Another option may be to switch to a lower dosage of a more potent statin. Glueck et $\mathrm{al}^{47}$ designed a study to evaluate the effectiveness and safety profile of rosuvastatin at doses of 5 and $10 \mathrm{mg} /$ day in consecutively referred patients with primary high LDL-C who were unable to tolerate other statins because of myalgia. This was a prospective, open-label pilot study enrolling 61 male and female patients aged 38 to 80 years with primary high LDL-C (mean $177 \mathrm{mg} / \mathrm{dL}$ ). After prescribing diet, rosuvastatin $5 \mathrm{mg}$ /day was administered to patients categorized by the National Cholesterol Education Program Adult Treatment Panel (NCEP ATP III) risk stratification as moderately high risk, and rosuvastatin $10 \mathrm{mg} /$ day was administered to patients categorized as high or very high risk. After treatment of over 36 weeks, rosuvastatin 5 and $10 \mathrm{mg}$ were associated with a LDL-C reduction of $42 \%$ and $39 \%$, respectively $(P<0.001$ vs baseline). Of the 61 patients, one receiving the $10-\mathrm{mg}$ dose discontinued rosuvastatin treatment because of unilateral muscular pain after 4 weeks; no significant aminotransferase or CK elevation was seen.

\section{Alternate-day statin dosing}

Several studies have evaluated non-approved statin dosing regimens in patients with SIM. Some have tested rapidly metabolized statins, such as lovastatin ${ }^{48,49}$ fluvastatin, ${ }^{50}$ or simvastatin. ${ }^{51}$ However, this approach has been based on the concept that statins with a longer half-life may maintain their lipid lowering effect over a longer period of time. For example, atorvastatin has a mean terminal half-life of $14 \mathrm{~h}$ and generates two active (orthohydroxy and parahydroxy) metabolites. $^{52}$ These metabolites contribute to $70 \%$ of its HMG-CoA reductase activity and have a half-life of $20-30$ h. $^{53}$ This justifies the use of atorvastatin in alternate-day dosage as it maintains its cholesterol lowering efficacy for longer.

Two studies have evaluated the potentiality of this approach in noncontrolled trials. In the first study, ${ }^{54}$ 61 patients with hypercholesterolemia received atorvastatin (10 mg) every other day before bedtime. After 8 weeks of treatment, total cholesterol and LDL-C were lowered by $23 \%$ and $30 \%$, respectively, and total triglycerides were reduced by $8 \%$; the increase in high density lipoprotein-cholesterol (HDL-C) level was not statistically significant. For the second study, ${ }^{55} 25$ patients with moderate hypercholesterolemia were treated with every-other-day administration of either atorvastatin (mean dose, $18.8 \mathrm{mg}$ ) or rosuvastatin (mean dose, $9.7 \mathrm{mg}$ ). With atorvastatin LDL-C decreased by $43 \%$, total triglycerides by $22 \%$, and HDL-C cholesterol increased by 
$9 \%$ (n $=9 ; P<0.05$ for all), while with rosuvastatin, LDL-C decreased by $28 \%$, total triglycerides by $15 \%$ and HDL-C increased by $10 \%(\mathrm{n}=16 ; P<0.05$ for all $)$.

Three other studies employed randomized, controlled designs. Matalka et $\mathrm{al}^{56}$ reported the results of the Alternate Day versus Daily Dosing of Atorvastatin study (ADDAS). This was a comparative efficacy and cost-effectiveness study in 35 patients randomly assigned to receive $10 \mathrm{mg}$ of atorvastatin daily or on alternate days. Dosage was adjusted to achieve the NCEP ATP III targets. Authors reported a 35\% reduction in LDL-C with $20 \mathrm{mg}$ every other day (mean dose $18 \mathrm{mg}$ ) compared with $38 \%$ reduction with $10 \mathrm{mg}$ every day (mean dosage $12 \mathrm{mg} /$ day). This difference was not statistically significant.

Jafari et $\mathrm{al}^{57}$ investigated 54 patients with LDL-C ranging 100-200 mg/dL. These patients were randomized into three atorvastatin dose groups: $10 \mathrm{mg}$ every day, $10 \mathrm{mg}$ every other day, and $20 \mathrm{mg}$ every other day. After 6 weeks of treatment, all three regimens significantly reduced total and LDL-C compared to baseline and more importantly, no statistically significant differences existed between the three groups. All regimens were well tolerated and none of the patients had a significant elevation of liver enzymes or CK during the course of the study. In a more recent study, ${ }^{58}$ 61 patients with LDL-C levels above $130 \mathrm{mg} / \mathrm{dL}$ were randomized to receive $20 \mathrm{mg}$ atorvastatin every day or every other day. In the every-other-day treatment group, there was a $36.1 \%$ reduction in LDL-cholesterol levels by the end of the first month $(P<0.01)$. At the end of 3 months there was a further decrease of $10.2 \%$ in LDL-cholesterol levels when compared to the 1 month levels $(P>0.05)$. The LDL-C levels of the group receiving $20 \mathrm{mg}$ atorvastatin every day was reduced by $41 \%$ by the end of 1 month $(P<0.01)$. At the end of 3 months, the difference between the changes in the all lipid parameters of the two groups was not found to be of statistical significance. Interestingly, both regimens significantly decreased high sensitivity-CRP and this effect was not different between the two treatment arms.

Ferrer-Garcia et $\mathrm{al}^{59}$ considered the alternate-day approach in patients with type 2 diabetes mellitus. They enrolled 41 patients that were assigned to receive $10 \mathrm{mg}$ atorvastatin as an initial dose every day until they reached the ATP III recommended LDL-C target $(<100 \mathrm{mg} / \mathrm{dL})$. After achieving LDL $<100 \mathrm{mg} / \mathrm{dL}$, the patients were assigned to the corresponding atorvastatin dose every other day for 12 weeks. Thirty-three patients correctly completed the study. LDL-C decreased 39\% after the everyday period and 23\% after the alternate-day atorvastatin dosing period $(P<0.05)$.
The target LDL-C concentration of $<100 \mathrm{mg} / \mathrm{dL}$ was maintained in 19 patients $(57.6 \%)$ during the alternate-day period. None of the 33 patients showed elevations in liver enzymes or CK during the alternate-day dosing period.

Rosuvastatin, the other statin that exhibits a long halflife (19 h), has also been employed in trials testing the infrequent statin dosing regimen. Mackie et a ${ }^{60}$ described two patients who were unable to tolerate daily atorvastatin therapy secondary to myalgias and were subsequently treated with rosuvastatin administered on Mondays, Wednesdays, and Fridays, at 2.5 and $5 \mathrm{mg}$, respectively. After 6 weeks, LDL-C was reduced by $38 \%$ and $20 \%$, respectively with the resolution of adverse effects. In a retrospective analysis of clinical charts at two lipid clinics, ${ }^{61} 51$ patients that had experienced statin intolerance were found to be treated with every-other-day rosuvastatin (mean dose $5.6 \mathrm{mg}$ ). The authors reported that $72.5 \%$ (37 out of 51) of patients were able to tolerate this regimen for 4 months. Mean LDL-C decreased by $34.5 \%(P<0.001)$ in patients who tolerated the regimen, enabling approximately $50 \%$ to achieve their LDL-C goal. Among patients treated with the every-other-day regimen, $27.5 \%$ (14 out of 51) re-experienced the symptoms of their prior statin intolerance.

In a 8-week, randomized, open-label, parallel trial, Wongwiwatthananukit et $\mathrm{al}^{62}$ evaluated the effect of $10 \mathrm{mg}$ rosuvastatin administered once daily or every other day in 81 patients with primary hypercholesterolemia. Among the 76 patients that completed the study, LDL-C levels were reduced by $48 \%$ and $39 \%$ in the once-daily and every-other-day groups, respectively $(P=0.011)$. The percentage of patients who achieved LDL-C goals according to NCEP ATP III guidelines was not significantly different between the once-daily (85\%) and every-other-day (70\%) groups $(P=0.180)$. In addition, both regimens were well tolerated, with no patient developing an elevation of more than 3 times baseline levels of aspartate aminotransferase or alanine aminotransferase or 10 times that of $\mathrm{CK}$.

Gadarla et al $^{63}$ also utilized rosuvastatin, at doses of 5 and $10 \mathrm{mg}$ twice weekly (Monday and Thursday) for a time longer than 3 weeks, in patients (mean age $62 \pm 8$ years) with SIM related to other lipid-lowering therapy (other statins or niacin or fibrate or combinations of these). This choice was well tolerated by $80 \%$ of patients and produced a significant $26 \%$ reduction of LDL-C from baseline.

Backes et $\mathrm{l}^{64}$ described eight patients with previous intolerance to daily statin dosing and examined their responses to once-weekly rosuvastatin therapy $(5-20 \mathrm{mg})$. Overall, this small group experienced a mean LDL-C reduction of 
$29 \%$. Possible explanations for tolerance according to the once-weekly regimen may include lower overall plasma concentrations from the less frequent dosing and the psychological factor of receiving only a once-weekly dose.

A larger study with similar dosing protocol was carried out by Ruisinger et $\mathrm{al}^{65}$ who enrolled 50 patients with a previous statin adverse event. Rosuvastatin once per week was tolerated by 37 (74\%) of the 50 study participants, with doses ranging from 2.5 to $20 \mathrm{mg}$ a week (mean $10 \mathrm{mg}$ ). Patients tolerating the once-a-week regimen experienced a $17 \%$ reduction in total cholesterol, $23 \%$ reduction in LDL-C, $12 \%$ reduction in triglycerides, and a $5 \%$ increase in highdensity lipoprotein cholesterol (all $P<0.001$ ), during a mean follow-up of 4 months.

Although the alternate-day statin dose administration has been demonstrated to be feasible and effective, some limitations must be stressed. First of all, it must be pointed out that this regimen allows a lower LDL-C reduction (up to $10 \%-15 \%$ less) compared to the every day regimen. This must be carefully considered in light of the ideal LDL-C target for each patient. Furthermore, these dosing regimens have not been proven to reduce cardiovascular events. Therefore, they must be always be regarded as alternative treatment options in well-selected patients such as those who did not tolerate more than one statin, even at lower dosage, and may otherwise go without any benefits from statin therapy.

\section{Combination therapy with infrequent statin dosing}

Athyros et $\mathrm{al}^{66}$ investigated a novel approach to managing statin intolerant patients based on the use of combination therapy where a statin was administered with an alternate dosing. They initially started 56 statin-intolerant patients on ezetimibe (10 mg/day). After evaluating the patients' responses, they added atorvastatin, $10 \mathrm{mg}$ twice weekly. Only $9 \%$ of the patients achieved their LDL-C goal on monotherapy, but $84 \%$ achieved the NCEP ATP III LDL-C target levels with the combination, which lowered LDL-C by $34 \%$.

\section{Nonstatin lipid-lowering drugs alone or in combination}

If myopathy recurs after trials with both multiple statins and a variety of dosing regimens, then nonstatin lipid lowering agents should be considered. These drugs include a bile acid sequestrant (colesevelam), an intestinal cholesterol absorption inhibitor (ezetimibe), fibrates, and niacin used alone or in combination.
Colesevelam is a nonabsorbable engineered polymer (water soluble hydrogel), which binds anionic and hydrophobic bile acids with higher affinity than cholestyramine. Dosage of $2-4 \mathrm{~g} /$ day results in a $15 \%-19 \%$ reduction of LDL-C. ${ }^{67}$ Conversely, ezetimibe is a specific inhibitor of intestinal cholesterol absorption through blocking the activity of NPC1L1 transporter and at the dosage of $10 \mathrm{mg} /$ day, is able to decrease LDL-C by $15 \%-20 \%{ }^{68}$ Colesevelam shows a better intestinal tolerability than cholestyramine and ezetimibe has no relevant side effects. Based on their safety profiles, these drugs may be used as monotherapy or in combination in the management of statin-intolerant patients. Gazi et al ${ }^{69}$ reported that among 27 statin-intolerant patients who started treatment with ezetimibe monotherapy, 25 completed three months of therapy showing a reduction of total and LDL-cholesterol of $18 \%$ and $26 \%$, respectively $(P<0.001$ for both). In another retrospective review of 16 statin-intolerant patients with diabetes mellitus or metabolic syndrome, Rivers et $\mathrm{al}^{70}$ reported that the combination of ezetimibe (10 mg/day) plus colesevelam (1.875 g twice daily) was well tolerated and determined a marked reduction of both LDL-C (42.2\%), and non-HDL-C (37.1\%). Fibrates have been reported to be very effective in patients with atherogenic dyslipidemia (high triglycerides and low HDL). ${ }^{71}$ McKenney et $\mathrm{al}^{72}$ examined the safety and efficacy of long-term coadministration of fenofibrate and ezetimibe in patients with mixed hyperlipidemia. In this study, the baseline total cholesterol was $262 \mathrm{mg} / \mathrm{dL}$, HDL-C $42 \mathrm{mg} / \mathrm{dL}$, LDL-C $162 \mathrm{mg} / \mathrm{dL}$, and triglyceride level $276 \mathrm{mg} / \mathrm{dL}$. There was a reduction in LDL-C by $20 \%$, non-HDL-C by $30 \%$, and apolipoprotein B by $26 \%$ on the combination therapy. All levels were significantly reduced compared with either monotherapy.

Niacin alone or associated with laropiprant has been reported to be effective in reducing LDL-C, total triglycerides, and increasing HDL, thus making it a possible alterative in patients with dyslipidemia. ${ }^{73}$ At present no data are available in the literature on the use of nicotinic acid in statin intolerant patients. Nevertheless, it must be noted that randomized controlled trials carried out in the pre-statin era clearly demonstrated that niacin was beneficial in preventing coronary events. ${ }^{74}$ Additional studies further demonstrated that this drug in combination with bile acid sequestrants was also able to slow the progression of coronary lesions. ${ }^{75,76}$ Very recently, two meta-analyses reviewed the effects of niacin alone or in combination on all cardiovascular events and on atherosclerosis in a total sample of about 6500 patients. ${ }^{77,78}$ These studies consistently reported that niacin 
significantly reduced major coronary events by $25 \%$, stroke by $26 \%$, and any cardiovascular events by $27 \%$. Moreover, when the effect of niacin on atherosclerosis evolution was evaluated, ${ }^{78}$ it was found that in comparison with the nonniacin group, more patients in the niacin group experienced regression of coronary atherosclerosis ( $92 \%$ relative increase) whereas the rate of patients with progression decreased by $41 \%$. Similar effects of niacin were found on carotid intimamedia thickness with a weighted mean difference in annual change of $-17 \mu \mathrm{m} /$ year.

\section{Dietary manipulation and lipid lowering nutraceuticals}

Strengthening the efficacy of lipid-lowering dietary interventions may be a reasonable consideration in patients who cannot take statins. This may be obtained by combining dietary ingredients showing cholesterol-lowering properties such as foods low in saturated fat and high in viscous fibers (eg, oats and barley), plant sterols, vegetable protein foods (soy), and nuts (eg, almonds). Jenkins et al ${ }^{79}$ evaluated the efficacy of this dietary portfolio versus lovastatin in 46 hyperlipemic adults. Participants were randomly assigned to a diet very low in saturated fat, based on whole-wheat cereals and low-fat dairy foods (control); the same diet plus lovastatin, $20 \mathrm{mg} / \mathrm{d}$ (statin); or a diet high in plant sterols $(1.0 \mathrm{~g} / 1000 \mathrm{kcal})$, soy protein (21.4 g/1000 kcal), viscous fibers $(9.8 \mathrm{~g} / 1000 \mathrm{kcal})$, and almonds (14 g/1000 kcal) (dietary portfolio). After four weeks, changes in LDL-C were 8.0\% ( $P=0.002), 30.9 \%$ $(P<0.001)$, and 28.6\% $(P<0.001)$, in the control, statin, and portfolio diets, respectively. It is important to note that the dietary portfolio led to a cholesterol reduction that was comparable to that observed with statin. Unfortunately, a limitation of this dietary formulation is the palatability. In fact, only $40 \%$ of participants to the Jenkins's trial found the dietary portfolio acceptable while the remaining thought that a greater variety of foods was required.

Dietary supplementation with nutraceuticals showing lipid-lowering effects may be an alternative option. Among these compounds, the yeast rice is of particular interest. Chinese red yeast rice is a dietary supplement made by fermenting the yeast, Monascus purpureus, over rice. Monascus yeast produces a family of substances called monacolins capable of inhibiting the enzyme HMG-CoA reductase and also contains unsaturated fatty acids and phytosterols. ${ }^{80}$ Becker et al ${ }^{81}$ evaluated this supplement to control hypercholesterolemia in statin-intolerant patients. They randomly assigned 62 patients with dyslipidemia (baseline LDL-C $163.3 \mathrm{mg} / \mathrm{dL}$ ) to receive red yeast rice, $1800 \mathrm{mg}$ (31 patients), or placebo (31 patients) twice daily for 24 weeks. All patients were concomitantly enrolled in a therapeutic lifestyle change program. Red yeast rice and therapeutic lifestyle change decreased LDL-C level by $27.3 \%$ after 12 weeks and this reduction was maintained after 24 weeks of therapy (-21.3\%). No increases in CK or pain levels were seen. In another study, ${ }^{82} 43$ patients with dyslipidemia and a history of statin discontinuation for myalgia were randomly assigned to red yeast rice $2400 \mathrm{mg}$ twice daily or pravastatin $20 \mathrm{mg}$ twice daily for 12 weeks. The LDL-C level decreased $30 \%$ in the red yeast rice group and $27 \%$ in the pravastatin group. A more recent report ${ }^{83}$ describes the results retrospectively observed in 25 patients who underwent treatment with red yeast rice for more than 4 weeks due to intolerance to lipid lowering medications. In those unable to tolerate daily statin use, the total cholesterol decreased 13\% and LDL-C decreased 19\% $(P<0.001$ compared to baseline).

One limitation of this therapeutic strategy is related to the fact that the LDL-C lowering potency of yeast rice is modest (ranging up to $20 \%$ ) so that its use can be proposed only in low-risk individuals or in those whom LDL-C is not far from the target. The prescription of combinations of different lipid-lowering nutraceuticals may be a way to increase the LDL-lowering efficacy of this approach. Cicero et $\mathrm{al}^{84}$ assessed the long-term effectiveness of daily administration of a combination of $2 \mathrm{~g}$ phytostanols, $500 \mathrm{mg}$ berberine, and $3 \mathrm{mg}$ monacoline in 48 statin-intolerant hypercholesterolemic patients. After 3 months, LDL-C was significantly decreased by $31 \%$ and total triglycerides by $16 \%$; more importantly, these effects were maintained up to the 1-year follow-up.

Although these results are intriguing, several aspects must be considered. The cholesterol-lowering effect of red yeast rice is due to monacolin $\mathrm{K}$, a natural form of lovastatin. The monacolin $\mathrm{K}$ content can vary widely between brands, and most patients purchase this supplement without verification of the monacolin $\mathrm{K}$ content. In addition, monacolins cannot be used as a dietary supplement at the dosage employed in the reported trials in some countries. Finally, there is no direct evidence that the use of nutraceuticals may produce any benefits in terms of cardiovascular prevention. Therefore, we agree with the concept that these agents should be considered as a fourth line approach, ${ }^{7}$ only after patients have demonstrated intolerance to multiple statins used at lowerthan-recommended doses, after using infrequent statin dosing protocol or after trials with nonstatins drugs. 


\section{Specific treatments of statin- induced myopathy}

There is limited experience concerning specific treatments of SIM. As it has been reported that SIM may be due to the statin-caused reduction of CoQ10. The CoQ10 supplementation has been proposed for the treatment of SIM. A successful trial with CoQ10 supplementation was reported by Caso et $a 1,{ }^{85}$ who assessed in a double-blinded protocol the effect of CoQ10 (100 mg/d, $n=18)$ or vitamin E (400 IU/d, $n=14)$ for 30 days in patients with myopathic symptoms. Pain severity decreased by $40 \%(P<0.001)$ and pain interference with daily activities decreased by $38 \%(P<0.02)$ in the group treated with CoQ10. In contrast, no changes in pain severity $(9 \%, P=$ not significant) or pain interference with daily activities $(11 \%, P=$ not significant) were observed with vitamin $\mathrm{E}$. These results suggest that CoQ10 supplementation may decrease muscle pain associated with statin treatment. However, these findings have not been replicated in larger trials and some concerns have been raised about the role of CoQ10 deficiency in the pathogenesis of SIM. ${ }^{18}$ Therefore, at present, the routine use of CoQ10 is not recommended in statin-intolerant patients.

It is well-known that vitamin deficiency is associated with myalgia and poor muscle function, ${ }^{86}$ which has led to the exploration of the potential benefits of vitamin supplementation in SIM. In a recent trial, 38 patients with statinrelated myalgia and vitamin D levels below $32 \mathrm{ng} / \mathrm{mL}$ were treated with vitamin D supplementation. ${ }^{87}$ After completing 3 months of vitamin D supplementation, the mean vitamin D levels increased and $92 \%$ of patients became myalgia free. This trial has several limitations, including its small study size and lack of a placebo group. These results, although interesting, need to be validated by a large randomized, double-blind, placebo-controlled trial.

In summary, even though these strategies have produced stimulating results, no specific recommendations can be issued concerning specific treatment to alleviate symptoms in patients presenting myalgia or myositis related to statin therapy.

\section{Conclusion}

In statin-intolerant patients the first step is to rule out any secondary causes of myopathy or liver toxicity (physical activity, hypothyroidism, drug interactions, related diseases). Secondly, it is important to determine whether the adverse effects are indeed related to statin therapy by statin dechallenge and rechallenge. If symptoms do not resolve, it is advisable to restart with the same statin at a lower dosage or to switch to another statin. The selection of the new statins should favor molecules metabolized via different pathways. In patients with a recurrence of symptoms, different approaches should be considered. Several small studies assessing unconventional dosing (every-other-day or weekly administration) of statins with longer half-life have been published. Although these studies presented encouraging alternatives for statin-intolerant patients, it has not been established that the cardiac risk reduction would be the same as with daily dosing regimens. Very few studies have evaluated the potential use of nonstatin lipid-lowering drugs (ezetimibe, bile acids sequestrants, and fibrates) alone or in combination in statin-intolerant patients. Therefore these approaches must be limited to selected patients after careful consideration of individual global cardiovascular and lipid targets. Finally, in low-risk individuals the use of herbal supplements effective in reducing LDL-C can be considered. At present, no specific recommendations can be issued regardig the use of these treatments for statin-induced myopathy.

\section{Disclosure}

MA has received research grants, speaker honoraria, and consultation fees from MSD, Pfizer, and Astra Zeneca.

\section{References}

1. Baigent C, Keech A, Kearney PM, et al; Cholesterol Treatment Trialists' (CTT) Collaborators. Cholesterol Treatment Trialists (CTT) Collaborators: efficacy and safety of cholesterol-lowering treatment: prospective meta-analysis of data from 90.056 participants in 14 randomized trials of statins. Lancet. 2005;366(9493):1267-1278.

2. Armitage J. The safety of statins in clinical practice. Lancet. 2007; 370(9601):1781-1790.

3. Gotto AMG. Statins, cardiovascular disease, and drug safety. Am J Cardiol. 2006;97(8A):3C-5C.

4. McKenney JM, Davidson MH, Jacobson TA, Guyton JR. Final conclusions and recommendations of the National Lipid Association Statin Safety Assessment Task Force. Am J Cardiol. 2006; 97(8A):89C-94C

5. Silva MA, Swanson AC, Gandhi PJ, Tataronis GR. Statin-related adverse events: a meta-analysis. Clin Ther. 2006;28(1):26-35.

6. Law M, Rudnicka AR. Statin safety: a systematic review. Am J Cardiol. 2006;97(8A):52C-60C.

7. Harper CR, Jacobson TA. Evidence-based management of statin myopathy. Curr Atheroscler Rep. 2010;12(5):322-330.

8. Davidson M, Clark J, Glass L, Kanumalla A. Statin safety: an appraisal from the adverse event reporting system. Am J Cardiol. 2006; 97(Suppl 1):32C-43C.

9. Bruckert E, Hayem G, Dejager S, Yau C, Begaud B. Mild to moderate muscular symptoms with high-dosage statin therapy in hyperlipidemic patients - the PRIMO study. Cardiovasc Drugs Ther. 2005; 19(6):403-414.

10. Shanahan RL, Kerzee JA, Sandhoff BG, Carroll NM, Merenich JA. Low myopathy rates associated with statins as monotherapy or combination therapy with interacting drugs in a group model health maintenance organization. Pharmacotherapy. 2005;25(3):345-351.

11. Harper CR, Jacobson TA. The broad spectrum of statin myopathy: from myalgia to rhabdomyolysis. Curr Opin Lipidol. 2007;18(4):401-408. 
12. Toth PP, Harper CR, Jacobson TA. Clinical characterization and molecular mechanisms of statin myopathy. Expert Rev Cardiovasc Ther. 2008;6(7):955-969.

13. Molokhia M, McKeigue P, Curcin V, Majeed A. Statin induced myopathy and myalgia: time trend analysis and comparison of risk associated with statin class from 1991-2006. PLoS ONE. 2008;3(6):e2522.

14. Newman C, Tsai J, Szarek M, Luo D, Gibson E. Comparative safety of atorvastatin $80 \mathrm{mg}$ versus $10 \mathrm{mg}$ derived from analysis of 49 completed trials in 14,236 patients. Am J Cardiol. 2006;97(1):61-67.

15. Wiviott SD, Cannon CP, Morrow DA, Ray KK, Pfeffer MA, Braunwald E. Can low-density lipoprotein be too low? The safety and efficacy of achieving very low low-density lipoprotein with intensive statin therapy: a PROVE IT-TIMI 22 substudy. J Am Coll Cardiol. 2005;46(8):1411-1416.

16. Davidson MH, Robinson JG. Safety of aggressive lipid management. J Am Coll Cardiol. 2007;49(17):1753-1762.

17. Hodel C. Myopathy and rhabdomyolysis with lipid-lowering drugs. Toxicol Lett. 2002;128(1-3):159-168.

18. Marcoff L, Thompson PD. The role of coenzyme Q10 in statin associated myopathy: a systematic review. J Am Coll Cardiol. 2007; 49(23):2231-2237.

19. Flint OP, Masters BA, Gregg RE, Durham SK. Inhibition of cholesterol synthesis by squalene synthase inhibitors does not induce myotoxicity in vitro. Toxicol Appl Pharmacol. 1997;145(1):91-98.

20. Laaksonen R, Jokelainen K, Sahi T, Tikkanen MJ, Himberg JJ. Decreases in serum ubiquinone concentrations do not result in reduced levels in muscle tissue during short-term simvastatin treatment in humans. Clin Pharmacol Ther. 1995;57(1):62-66.

21. Dirks AJ, Jones KM. Statin-induced apoptosis and skeletal myopathy. Am J Physiol Cell Physiol. 2006;291(6):C1208-C1212.

22. Guijarro C,Blanco-Colio LM, Ortego M, etal.3-Hydroxy-3-methylglutaryl coenzyme a reductase and isoprenylation inhibitors induce apoptosis of vascular smooth muscle cells in culture. Circ Res. 1998 83(5):490-500.

23. Mohaupt MG, Karas RH, Babiychuk EB, et al. Association between statin-associated myopathy and skeletal muscle damage. Can Med Assoc J. 2009;181(1-2):E11-E18.

24. Sirvent P, Mercier J, Vassort G, Lacampagne A. Simvastatin triggers mitochondria-induced $\mathrm{Ca} 2+$ signaling alteration in skeletal muscle. Biochem Biophys Res Commun. 2005;329(3):1067-1075.

25. Group SC, Link E, Parish S, et al. SLCO1B1 variants and statininduced myopathy - a genome wide study. $N$ Engl J Med. 2008; 359(8):789-799.

26. Cash J, Callender ME, McDougall NI, Young IS, Nicholls DP. Statin safety and chronic liver disease. Int J Clin Pract. 2008;62(12):1831-1835.

27. Cohen D, Anania F, Chalasani N. An assessment of statin safety by hepatologists. Am J Cardiol. 2006;97(8A):77C-81C.

28. Pasternak RC, Smith SC Jr, Bairey-Merz CN, Grundy SM, Cleeman JI, Lenfant C. ACC/AHA/NHLBI clinical advisory on the use and safety of statins. Circulation. 2002;106(8):1024-1028.

29. Argo CK, Loria P, Caldwell SH, Lonardo A. Statins in liver disease: a molehill, an iceberg, or neither? Hepatology. 2008;48(2):662-669.

30. Calderon RM, Luigi X, Cubeddu LX, Goldberg RB, Schiff ER. Statins in the treatment of dyslipidemia in the presence of elevated liver aminotransferase levels: a therapeutic dilemma. Mayo Clin Proc. 2010;85(4):349-356.

31. De Denus S, Spinler SA, Miller K, Peterson AM. Statins and liver toxicity: a meta-analysis. Pharmacotherapy. 2004;24(5):584-591.

32. Kornbrust DJ, MacDonald JS, Peter CP, et al. Toxicity of the HMGcoenzyme A reductase inhibitor, lovastatin, to rabbits. J Pharmacol Exp Ther. 1989;248(2):498-505.

33. Dale KM, White CM, Henyan NN, Kluger J, Coleman CI. Impact of statin dosing intensity on transaminase and creatine kinase. Am J Med. 2007;120(8):706-712.

34. Pasanen MK, Fredrikson H, Neuvonen PJ, Niemi M. Different effects of SLCO1B1 polymorphism on the pharmacokinetics of atorvastatin and rosuvastatin. Clin Pharmacol Ther. 2007;82(6):726-733.
35. Chalasani N. Statins and hepatotoxicity: focus on patients with fatty liver. Hepatology. 2005;41(4):690-695.

36. Athyros VG, Tziomalos K, Gossios TD, et al; GREACE Study Collaborative Group. Safety and efficacy of long-term statin treatment for cardiovascular events in patients with coronary heart disease and abnormal liver tests in the Greek Atorvastatin and Coronary Heart Disease Evaluation (GREACE) Study: a post-hoc analysis. Lancet. 2010;376(9756):1916-1922.

37. Tolman KG. The liver and lovastatin. Am J Cardiol. 2002;89(12): $1374-1380$

38. Cziraky MJ, Willey VJ, McKenney JM. Statin safety: an assessment using an administrative claims database. Am J Cardiol. 2006; 97(8A):61C-68C.

39. Bhardwaj SS, Chalasani N. Lipid lowering agents that cause drug induced hepatotoxicity. Clin Liver Dis. 2007;11(3):597-613.

40. Clarke AT, Mills PR. Atorvastatin associated liver disease. Dig Liver Dis. 2006;38(10):772-777.

41. Castiella A, Fernandez J, Zapata E. Autoimmune hepatitis after treatment with fluvastatin. Liver Int. 2007;27(4):592.

42. Wolters LM, Van Buuren HR. Rosuvastatin-associated hepatitis with autoimmune features. Eur J Gastroenterol Hepatol. 2005;17(5): 589-590.

43. Pelli N, Setti M. Atorvastatin as a trigger of autoimmune hepatitis. J Hepatol. 2004;40(4):716.

44. Grattagliano I, Portincasa P, Palmieri VO, Palasciano G. Overview on the mechanisms of drug-induced liver cell death. Ann Hepatol. 2002; 1(4):162-168.

45. Anfossi G, Massucco P, Bonomo K, Trovati M. Prescription of statins to dyslipidemic patients affected by liver diseases: a subtle balance between risks and benefits. Nutr Metab Cardiovasc Dis. 2004; 14(4):215-224.

46. Hansen KE, Hildebrand JP, Ferguson EE, Stein JH. Outcomes in 45 patients with statin-associated myopathy. Arch Intern Med. 2005; 165(22):2671-2676.

47. Glueck CJ, Aregawi D, Agloria M, et al. Rosuvastatin 5 and $10 \mathrm{mg} / \mathrm{d}$ : a pilot study of the effects in hypercholesterolemic adults unable to tolerate other statins and reach LDL cholesterol goals with nonstatin lipid-lowering therapies. Clin Ther. 2006;28(6):933-942.

48. Rindone JP, Achacoso R, Bledsoe R. Effect of lovastatin administered every other day on serum low density ipoprotein cholesterol $>160 \mathrm{mg} / \mathrm{dl}$. Am J Cardiol. 1995;76(4):312-313.

49. Dennis VC, Britton ML, Sirmans SM, Letassy NA, Freeman DA. The use of alternate-day lovastatin in hypercholesterolemic men. Ann Pharmacother. 1997;31(6):708-712.

50. Rindone JP, Hiller D, Arriola G. A comparison of fluvastatin $40 \mathrm{mg}$ every other day versus $20 \mathrm{mg}$ every day in patients with hypercholesterolemia. Pharmacotherapy. 1998;18(4):836-839.

51. Copher HR, Steward RD. Daily dosing versus alternate-dosing of simvastatin in patients with hypercholesterolemia. Pharmacotherapy. 2002;22(9):1110-1116.

52. Lennerna SH. Clinical pharmacokinetics of atorvastatin. Clin Pharmacokinet. 2003;42(13):1141-1160.

53. Lins RL, Matthys KE, Verpooten GA, et al. Pharmacokinetics of atorvastatin and its metabolites after single and multiple dosing in hypercholesterolaemic haemodialysis patients. Nephro Dial Transplant. 2003;18(5):967-976.

54. Piamsomboon C, Laothavorn P, Saguonwong S, Chatloong B, Nasawadi C, Tanprasert P. Efficacy and safety of atorvastatin $10 \mathrm{mg}$ every other day in hypercholesterolemia. J Med Assoc Thai. 2002; 85(3):297-300.

55. Juszczyk MA, Seip RL, Thompson PD. Decreasing LDL cholesterol and medication cost with every-other-day statin therapy. Prev Cardiol. 2005;8(4):197-199.

56. Matalka MS, Ravnan MC, Deedwania MD. Is alternate daily dose of atorvastatin effective in treating patients with hyperlipidemia? The Alternate Day versus Daily Dosing of Atrovastatin Study (ADDAS). Am Heart J. 2002;144(4):674-677. 
57. Jafari M, Ebrahimi R, Ahmadi-Kashani M, Balian H, Bashir M. Efficacy of alternate-day dosing versus daily dosing of atorvastatin. J Cardiovasc Pharmacol Ther. 2003;8(2):123-126.

58. Keles T, Akar Bayram N, Kayhan T, et al. The comparison of the effects of standard $20 \mathrm{mg}$ atorvastatin daily and $20 \mathrm{mg}$ atorvastatin every other day on serum LDL-cholesterol and high sensitive C-reactive protein levels. Anadolu Kardiyol Derg. 2008;8(6):407-412.

59. Ferrer-Garcia JC, Perez-Silvestre J, Martinez-Mir I, Herrera-Ballester A. Alternate-day dosing of atorvastatin: effects in treating type 2 diabetic patients with dyslipidemia. Acta Diabetol. 2006;43(3):75-78.

60. Mackie BD, Satija S, Nell C, Miller J, Sperling LS. Monday, Wednesday and Friday dosing of rosuvastatin in patients intolerant to statin therapy. Am J Cardiol. 2007;99(2):291.

61. Bakes JM, Vernero CV, Gibson CA, et al. Effectiveness and tolerability of every-other-day rosuvastatin dosing in patients with prior statin intolerance. Ann Pharmacother. 2008;42(3):341-346.

62. Wongwiwatthananukit S, Sansanayudh N, Dhummauppakorn R, Kitiyadisai C. Efficacy and safety of rosuvastatin every other day compared with once daily in patients with hypercholesterolemia. Ann Pharmacother. 2006;40(11):1917-1923.

63. Gadarla M, Kearns AK, Thompson PD. Efficacy of rosuvastatin ( $5 \mathrm{mg}$ and $10 \mathrm{mg}$ ) twice a week in patients intolerant to daily statins. $\mathrm{Am} \mathrm{J}$ Cardiol. 2008;101(12):1747-1748.

64. Backes JM, Moriarty PM, Ruisinger JF, Gibson CA. Effects of once weekly rosuvastatin among patients with a prior statin intolerance. Am J Cardiol. 2007;100(3):554-555.

65. Ruisinger JF, Backs JM, Gibson CA, Moriarty PM. Once a week rosuvastatin $(2.5$ to $20 \mathrm{mg}$ ) in patients with a previous statin intolerance. Am J Cardiol. 2009;103(3):393-394.

66. Athyros VG, Tziomalos K, Kakafika AI, Koumaras H, Karagiannis A, Mikhailidis DP. Effectiveness of ezetimibe alone or in combination with twice a week atorvastatin $(10 \mathrm{mg})$ for statin intolerant high-risk patients. Am J Cardiol. 2008;101(4):483-485.

67. Davidson MH, Donovan JM, Misir S, Jones MR. A 50-week extension study on the safety and efficacy of colesevelam in adults with primary hypercholesterolemia. Am J Cardiovasc Drugs. 2010;10(5):305-314.

68. Pandor A, Ara RM, Tumur I, et al. Ezetimibe monotherapy for cholesterol lowering in 2,722 people: systematic review and meta-analysis of randomized controlled trials. J Intern Med. 2009;265(5):568-580.

69. Gazi IF, Daskalopoulou SS, Nair DR, Mikhailidis DP. Effect of ezetimibe in patients who cannot tolerate statins or cannot get to the low density lipoprotein cholesterol target despite taking a statin. Curr Med Res Opin. 2007;23(9):2183-2192.

70. Rivers SM, Kane MP, Busch RS, Bakst G, Hamilton RA. Colesevelam hydrochloride-ezetimibe combination lipid-lowering therapy in patients with diabetes or metabolic syndrome and a history of statin intolerance. Endocr Pract. 2007;13(1):11-16.

71. Elam M, Lovato LC, Ginsberg H. Role of fibrates in cardiovascular disease prevention, the ACCORD-Lipid perspective. Curr Opin Lipidol. 2011;22(1):55-61.

72. McKenney JM, Farnier M, Lo KW, et al. Safety and effi cacy of longterm co-administration of fenofibrate and ezetimibe in patients with mixed hyperlipidemia. J Am Coll Cardiol. 2006;47(8):1584-1587.
73. Brooks EL, Kuvin JT, Karas RH. Niacin's role in the statin era. Expert Opin Pharmacother. 2010;11(14):2291-2300.

74. Canner PL, Berge KG, Wenger NK, et al. Fifteen year mortality in coronary drug project patients: long-term benefit with niacin. $\mathrm{J} \mathrm{Am}$ Coll Cardiol. 1986;8(6):1245-1255.

75. Blankenhorn DH, Nessim SA, Johnson RL, Sanmarco ME, Azen SP, Cashin-Hemphill L. Beneficial effects of combined colestipol-niacin therapy on coronary atherosclerosis and coronary venous bypass grafts. JAMA. 1987;257(23):3233-3240.

76. Brown G, Albers JJ, Fisher LD, et al. Regression of coronary artery disease as a result of intensive lipid-lowering therapy in men with high levels of apolipoprotein B. N Engl J Med. 1990;323(19):1289-1298.

77. Duggal JK, Singh M, Attri N, et al. Effect of niacin therapy on cardiovascular outcomes in patients with coronary artery disease. J Cardiovasc Pharmacol Ther. 2010;15(2):158-166.

78. Labreuche J, Deplanque D, Touboul PJ, Bruckert E, Amarenco P. Association between change in plasma triglyceride levels and risk of stroke and carotid atherosclerosis: systematic review and metaregression analysis. Atherosclerosis. 2010;212(1):9-15

79. Jenkins DJ, Kendall CW, Marchie A, et al. Effects of a dietary portfolio of cholesterol-lowering foods vs lovastatin on serum lipids and C-reactive protein. JAMA. 2003;290(4):502-510.

80. Ma J, Li Y, Ye Q, et al. Constituents of red yeast rice, a traditional Chinese food and medicine. J Agric Food Chem. 2000;48(11):5220-5225.

81. Becker DJ, Gordon RY, Halbert SC, French B, Morris PB, Rader D. Red yeast rice for dyslipidemia in statin-intolerant patients: a randomized trial. Ann Intern Med. 2009;150(12):830-839.

82. Halbert SC, French B, Gordon RY, et al. Tolerability of red yeast rice $(2,400 \mathrm{mg}$ twice daily) versus pravastatin (20 $\mathrm{mg}$ twice daily) in patients with previous statin intolerance. Am J Cardiol. 2010; 105(2):198-204.

83. Venero CV, Venero JV, Wortham DC, Thompson PT. Lipid-lowering efficacy of red yeast rice in a population intolerant to statins. $A m J$ Cardiol. 2010;105(5):664-666.

84. Cicero AFG, Derosa G, Bove M, Imola F, Borghi C, Gaddi AV. Long-term effectiveness and safety of a nutraceuticals-based approach to reduce cholesterolemia in statin intolerant subjects with and without metabolic syndrome. Curr Top Nutraceutical Res. 2009;(3-4):121-126.

85. Caso G, Kelly P, McNurlan MA, Lawson WE. Effect of coenzyme Q10 on myopathic symptoms in patients treated with statins. Am J Cardiol. 2007;99(10):1409-1412.

86. Erkal MZ, Wilde J, Bilgin Y, et al. High prevalence of vitamin D deficiency, secondary hyperparathyroidism and generalized bone pain in Turkish immigrants in Germany: identification of risk factors. Osteoporos Int. 2006;17(8):1133-1140.

87. Ahmed W, Khan N, Glueck CJ, et al. Low serum $25(\mathrm{OH})$ vitamin D levels $(<32 \mathrm{ng} / \mathrm{mL})$ are associated with reversible myositis myalgia in statin-treated patients. Transl Res. 2009;153(1):11-16.

Diabetes, Metabolic Syndrome and Obesity: Targets and Therapy

Dovepress

\section{Publish your work in this journal}

Diabetes, Metabolic Syndrome and Obesity: Targets and Therapy is an international, peer-reviewed open-access journal committed to the rapid publication of the latest laboratory and clinical findings in the fields of diabetes, metabolic syndrome and obesity research. Original research, review, case reports, hypothesis formation, expert opinion and commentaries are all considered for publication. The manuscript management system is completely online and includes a very quick and fair peer-review system, which is all easy to use. Visit http://www.dovepress.com/testimonials.php to read real quotes from published authors. 